\title{
COMPOSITION AND TOEPLITZ OPERATORS ON GENERAL DOMAINS
}

\author{
GUANGFU CAO
}

(Received November 26, 2002, revised July 26, 2004)

\begin{abstract}
Characterizations of invertible and Fredholm composition operators are obtained for Bergman spaces on connected domains in the complex plane. In addition, the isomorphism between Toeplitz algebras and their K-theory are discussed.
\end{abstract}

1. Introduction. Let $M$ and $N$ be two open, connected, non-empty subsets in the complex plane, which are said simply to be domains. Let $L^{2}(M)$ denote the space of complexvalued, measurable functions defined on $M$ that are square integrable with respect to the area measure on $M$. The Bergman space of $M$, denoted by $L_{a}^{2}(M)$, is by definition the set of analytic functions in the Hilbert space $L^{2}(M)$.

Denoting by $\partial M$ the topological boundary of $M$, a point $\lambda \in \partial M$ is said to be removable with respect to $L_{a}^{2}(M)$ if there exists an open neighborhood $V$ of $\lambda$ such that each function in $L_{a}^{2}(M)$ can be extended to an analytic function defined on $M \cup V$. We denote by $\partial_{r} M$ the set of points of $\partial M$ which are removable with respect to $L_{a}^{2}(M)$. The Bergman essential boundary of $M$, denoted by $\partial_{e} M$, is defined to be the set of points of $\partial M$ which are not removable with respect to $L_{a}^{2}(M)$, so that

$$
\partial_{e} M=\partial M-\partial_{r} M .
$$

If $M$ is finitely connected, that is, there are finite holes in $M$, then the Bergman removable boundary of $M$ is nothing but the set of isolated points of $\partial M$. The concept of essential boundary is first introduced in Axler, Conway and McDonald [1], where they proved that the essential spectrum of a Toeplitz operator with continuous symbol on a domain coincides with the range of the symbol on its essential boundary. In the present paper, we are concerned with two classes of operators on domains, namely, composition and Toeplitz operators.

In Sections 2 and 3 of this paper, we obtain characterizations of invertible and Fredholm composition operators. In the last section, we discuss the isomorphism between two Toeplitz algebras and their K-theory.

The author is very grateful to the referee for his many invaluable suggestions.

2. Invertibility of composition operators. The research on invertibility of composition operators on Hardy space $H^{2}(\boldsymbol{D})$ was initiated in [2]. Hatori [3] and Cao and Sun [4] then

2000 Mathematics Subject Classification. Primary 47B33; Secondary 19K99, 46L80.

Key words and phrases. Composition operator, Toeplitz operator, essential boundary.

Supported by National Natural Science Foundation of China. 
proved analogous results for the case of $H^{2}\left(B_{n}\right)$, where $B_{n}$ is the unit ball of the complex Euclidean $n$-space $C^{n}$. For composition operators on other spaces, similar results were proved by Bourdon [5] and Singh and Veluchamy [6]. Also, Takagi [7] studied the Fredholm weighted composition operators on $C(X)$, the algebra of complex-valued continuous functions defined on a compact Hausdorff space $X$, or $L^{p}(X)$, where $X$ is a set with some regular property like intervals or balls in the Euclidean $n$-space $\boldsymbol{R}^{n}$. In [8], Mihaila discussed the invertibility of composition operators on Riemann surfaces.

It is well-known that every composition operator on the Hardy space for one complex variable is bounded. However, a composition operator may be unbounded for general domains. For example, if

$$
M=\{z \in \boldsymbol{C}|| z \mid>1\}, \quad N=\{z \in \boldsymbol{C}|0<| z \mid<1\},
$$

and $\rho(z)=1 / z$ is the map from $M$ to $N, C_{\rho}$ is the composition operator from $L_{a}^{2}(N)$ to $L_{a}^{2}(M)$ which is defined by $C_{\rho} f=f \circ \rho$, then $C_{\rho}$ is an unbounded operator. In fact, for $f \in L_{a}^{2}(N), C_{\rho} f$ may not be even in $L_{a}^{2}(M)$. For instance, if $f(z)=z$, then $f \in L_{a}^{2}(N)$, but $C_{\rho} f \notin L_{a}^{2}(M)$.

From the example above, we also see that even if $\rho$ is invertible, $C_{\rho}$ may not be invertible.

Definition 1. Let $\mu$ be a positive measure on a domain $M$ in $\boldsymbol{C}$. $\mu$ is said to be a Carleson-type measure on $M$ if there exists a constant $C>0$ such that for any $f \in$ $L^{2}(M, d \mu)$

$$
\int_{M}|f|^{2} d \mu \leq C \int_{M}|f|^{2} d A
$$

It is obvious that $\mu$ is a Carlson-type measure if and only if there is a constant $C>0$ such that for an arbitrary measurable subset $E$ of $M$,

$$
\mu(E) \leq C A(E),
$$

where $A(E)$ is the Lebesgue measure of $E$.

Proposition 1. Suppose that $M$ and $N$ are domains in $\boldsymbol{C}$, and $\rho: M \rightarrow N$ is an analytic map such that $d A \circ \rho^{-1}$ is a Carleson-type measure. Then $C_{\rho}$ is a bounded operator from $L_{a}^{2}(N)$ to $L_{a}^{2}(M)$.

ProOF. By the identity

$$
\int_{N} f d A \circ \rho^{-1}=\int_{M} f \circ \rho d A, \quad f \in L_{a}^{2}(N)
$$

and the definition of a Carlson-type measure, one can see easily that $C_{\rho}$ is bounded.

THEOREM 2. Suppose that $M$ and $N$ are domains in $C$, and $\rho: M \rightarrow N$ is a nonconstant analytic map such that both $d A \circ \rho^{-1}$ and $d A \circ \rho$ are Carleson-type measures. Then $C_{\rho}$ is an invertible operator from $L_{a}^{2}(N)$ onto $L_{a}^{2}(M)$ if and only if

(i) $\rho$ is injective, and

(ii) $\quad N-\rho(M) \subset \partial_{r}(\rho M)$. 
Proof. Assuming that $C_{\rho}$ is an invertible operator from $L_{a}^{2}(N)$ onto $L_{a}^{2}(M)$, we first prove that $\rho$ is injective. Let $K_{z}^{M}(w)$ and $K_{z}^{N}(w)$ denote respectively the reproducing kernels of $L_{a}^{2}(M)$ and $L_{a}^{2}(N)$. It is routine to check that for any $z \in M$, we have

$$
C_{\rho}^{*} K_{z}=K_{\rho(z)} \cdot
$$

If there are $z_{1}, z_{2} \in M$ with $z_{1} \neq z_{2}$ such that $\rho\left(z_{1}\right)=\rho\left(z_{2}\right)$, then $C_{\rho}^{*}\left(K_{z_{1}}-K_{z_{2}}\right)=0$. It is obvious that $K_{z_{1}}-K_{z_{2}} \neq 0$, and hence this contradicts the invertibility of $C_{\rho}$. Hence $\rho$ must be injective. (i) is proved.

Since $\rho(M) \subset N$, we see that $L_{a}^{2}(N) \subset L_{a}^{2}(\rho(M)) . C_{\rho}$ is also an operator from $L_{a}^{2}(\rho(M))$ to $L_{a}^{2}(M)$, which we write by $\widetilde{C_{\rho}}$. Since $d A \circ \rho^{-1}$ is a Carlson-type measure on $N$, so is also on $\rho(M)$, it is verified that $\widetilde{C_{\rho}}$ is a bounded operator from $L_{a}^{2}(\rho(M))$ to $L_{a}^{2}(M)$ by Proposition 1 . This shows that $C_{\rho}^{-1} \circ \widetilde{C_{\rho}}$ is a bounded operator from $L_{a}^{2}(\rho(M))$ to $L_{a}^{2}(N)$, so that for any $g \in L_{a}^{2}(\rho(M))$, we have $C_{\rho}^{-1} \circ{\widetilde{C_{\rho}}}_{g} \in L_{a}^{2}(N)$. Let

$$
h=C_{\rho}^{-1} \circ \widetilde{C_{\rho}} g \in L_{a}^{2}(N),
$$

and $T_{\rho}=C_{\rho^{-1}}$ be the composition operator from $R\left(\widetilde{C_{\rho}}\right)$ to $L_{a}^{2}(\rho M)$. Then

$$
T_{\rho} C_{\rho} h=T_{\rho} \widetilde{C_{\rho}} g=g .
$$

For any $w \in \rho(M)$, it is easy to see that $\left(C_{\rho} h\right)\left(\rho^{-1} w\right)=g(w)$, and further $h(w)=g(w)$. Hence every function in $L_{a}^{2}(\rho(M))$ may be extended to a function in $L_{a}^{2}(N)$. This proves (ii).

Conversely, if (i) and (ii) in the Theorem hold, then $\widetilde{C_{\rho}}: L_{a}^{2}(\rho(M)) \rightarrow L_{a}^{2}(M)$ is invertible with inverse $\widetilde{C_{\rho^{-1}}}$. Since $N-\rho(M) \subset \partial_{r}(\rho(M))$, we see that $L_{a}^{2}(\rho(M))=L_{a}^{2}(N)$. This shows that $C_{\rho}$ is indeed invertible.

REMARK. If we assume only that $d A \circ \rho^{-1}$ is a Carleson-type measure, $C_{\rho}$ may not be an invertible operator even if $\rho$ is invertible. For instance, in the previous example, $d A \circ \rho$ is a Carlson-type measure, but $C_{\rho^{-1}}$ is not invertible.

EXAmple. Suppose that $\left\{\alpha_{i}\right\}_{i=1}^{\infty}$ is a Blaschke sequence in the unit disc $D$ of the complex plane, $M=D-\left\{\alpha_{i}\right\}_{i=1}^{\infty}, N=D$ and $\rho$ is the inclusion map from $M$ into $N$. Then it is obvious that $d A \circ \rho$ and $d A \circ \rho^{-1}$ are Carleson-type measures, $\rho$ satisfies the assumption of Theorem 2. Indeed, it is easy to see that $\left\{\alpha_{i}\right\}_{i=1}^{\infty}$ is the removable boundary of $\rho(M)$ by the uniqueness theorem of analytic functions. Furthermore, $C_{\rho}$ is an invertible operator from $L_{a}^{2}(N)$ onto $L_{a}^{2}(M)$.

COROLlary. Suppose that $M$ and $N$ are domains in $C$, and $\rho: M \rightarrow N$ is an analytic map such that $\partial_{r}(\rho(M))=\emptyset$. If both $d A \circ \rho^{-1}$ and $d A \circ \rho$ are Carlson-type measures, then $C_{\rho}$ is an invertible operator from $L_{a}^{2}(N)$ onto $L_{a}^{2}(M)$ if and only if $\rho$ is invertible.

REMARK. It is well-known that if $\rho$ is an analytic self-map of the unit disc $D$ in the complex plane, then $C_{\rho}$ is always a bounded linear operator on Bergman space $L_{a}^{2}(D)$ or Hardy space $H^{2}(D)$ and $C_{\rho}$ is invertible if and only if $\rho$ is invertible. By Theorem 2, we see that the invertibility of a composition operator is not equivalent to the invertibility of its 
symbol for general domains in the complex plane. However, if the topological boundary of the domain does not contain any removable points, then the invertibility of the composition operator does imply the invertibility of its symbol.

3. Fredholmness of composition operators. We know that on almost all spaces of analytic functions, the invertibility of a composition operator is equivalent to the property that being Fredholm. It is thus natural to study the Fredholmness of composition operators on general domains.

LEMma 3 (Axler, Conway and McDonald [9]). For any domain $G$ in $\boldsymbol{C}$, the area measure of $\partial_{r} G$ is zero.

THEOREM 4. Suppose that $M$ and $N$ are bounded domains in $\boldsymbol{C}$, and $\rho: M \rightarrow N$ is a nonconstant analytic map such that both $d A \circ \rho^{-1}$ and $d A \circ \rho$ are Carleson-type measures. Then $C_{\rho}$ is a Fredholm operator from $L_{a}^{2}(N)$ to $L_{a}^{2}(M)$ if and only if

(i) $\rho$ is injective, and

(ii) $\quad N-\rho(M) \subset \partial_{r}(\rho M)$.

Proof. By Theorem 2, we need only to prove the necessity. Assuming that $C_{\rho}$ is Fredholm, we first prove that $\rho$ is injective. Indeed, if $z_{1}, z_{2} \in M$ with $z_{1} \neq z_{2}$ such that $\rho\left(z_{1}\right)=\rho\left(z_{2}\right)$, then there exist open subsets $U_{1}, U_{2}$ of $M$ such that $z_{i} \in U_{i}, i=1,2$, and $U_{1} \cap U_{2}=\emptyset$. Write $w=\rho\left(z_{i}\right)$. Since $\rho$ is an open map, both $\rho\left(U_{1}\right)$ and $\rho\left(U_{2}\right)$ are open sets which contain $w$. Thus $\rho\left(U_{1}\right) \cap \rho\left(U_{2}\right)$ is a non-empty open subset of $N$. Choose a sequence $\left\{w_{k}\right\}$ in $\rho\left(U_{1}\right) \cap \rho\left(U_{2}\right)$ with $w_{k} \neq w_{k^{\prime}}$ for $k \neq k^{\prime}$. Then there are sequences $\left\{z_{k}^{(1)}\right\} \subset U_{1}$ and $\left\{z_{k}^{(2)}\right\} \subset U_{2}$ such that $\rho\left(z_{k}^{(i)}\right)=w_{k}$ for $i=1$, 2. Set $f_{k}=K_{z_{k}^{(1)}}-K_{z_{k}^{(2)}}$. Then $\left\{f_{k}\right\}$ is a linear independent sequence in $L_{a}^{2}(M)$, since $z_{k}^{(i)} \neq z_{k^{\prime}}^{(j)}$ for $k \neq k^{\prime}$ or $i \neq j$. However we have

$$
C_{\rho}^{*} f_{k}=K_{\rho\left(z_{k}^{(1)}\right)}-K_{\rho\left(z_{k}^{(2)}\right)}=0,
$$

which contradicts the Fredholmness of $C_{\rho}$. Hence $\rho$ is injective. (i) is proved.

It is obvious that $\widetilde{C_{\rho}}$ is a bounded operator from $L_{a}^{2}(\rho(M))$ to $L_{a}^{2}(M)$ by Proposition 1. Since $\rho(M) \subset N$, we have $L_{a}^{2}(N) \subset L_{a}^{2}(\rho(M))$. We show that $N \subset \overline{\rho(M)}$. Suppose not. Then there exist $w \in N$ and an open subset $U(w) \subset N$ which satisfies $U(w) \cap \rho(M)=\emptyset$. Set

$$
f_{k}(z)=\left(\frac{1}{z-w}\right)^{k}
$$

Then $\left\{f_{k}\right\} \subset L_{a}^{2}(\rho(M)), \quad k \geq 1$, is a linear independent sequence, and $f_{k} \notin L_{a}^{2}(N)$. However, $C_{\rho}\left(L_{a}^{2}(N)\right)$ is a closed subspace of $L_{a}^{2}(M)$, and

$$
\operatorname{dim} \frac{L_{a}^{2}(M)}{C_{\rho}\left(L_{a}^{2}(N)\right)}<\infty,
$$


since $d A \circ \rho$ is a Carleson-type measure. Hence we may easily see that $\widetilde{C_{\rho}}$ is an invertible operator from $L_{a}^{2}(\rho(M))$ onto $L_{a}^{2}(M)$. Thus

$$
\operatorname{dim} \frac{R\left(\widetilde{C_{\rho}}\right)}{R\left(C_{\rho}\right)}<\infty
$$

It is not difficult to check that $L_{a}^{2}(N)$ is a closed subspace of $L_{a}^{2}(\rho(M))$. Hence we see that

$$
\operatorname{dim} \frac{L_{a}^{2}(\rho(M))}{L_{a}^{2}(N)}<\infty
$$

since $\operatorname{dim} R\left(\widetilde{C_{\rho}}\right) / R\left(C_{\rho}\right)<\infty$. Denote by $\left[f_{i}\right]$ the class of $f_{i}$ in $L_{a}^{2}(\rho(M)) / L_{a}^{2}(N)$. If $\left\{\left[f_{i}\right]\right\}$ is linearly dependent, then there exist $\alpha_{i} \in \boldsymbol{C}, i=1, \ldots, k$ with $\alpha_{k} \neq 0$ such that

$$
g=\sum_{i=1}^{k} \alpha_{i} f_{i} \in L_{a}^{2}(N) .
$$

However, since $\left|f_{i}\right|(z) \rightarrow \infty$ as $z \rightarrow w$, this implies that $|g(z)| \rightarrow \infty$ as $z \rightarrow w$. This contradiction shows that $N \subset \overline{\rho(M)}$.

Now we prove that $N-\rho(M) \subset \partial_{r}(\rho(M))$. Let $f_{1}, f_{2}, \ldots, f_{n}$ be functions in $L_{a}^{2}(\rho(M))$ such that $\left\{\left[f_{i}\right]\right\}_{i=1}^{n}$ is a basis of $L_{a}^{2}(\rho(M)) / L_{a}^{2}(N)$. For every $1 \leq i \leq n$ and a non-negative integer $m, f_{i} z^{m}$ is in $L_{a}^{2}(\rho(M))$, since $N$ is a bounded domain and so is $\rho(M)$. Since $L_{a}^{2}(\rho(M)) / L_{a}^{2}(N)$ is of a finite dimension, $\left\{\left[f_{i} z^{m}\right]\right\}_{m=0}^{\infty}$ is linearly dependent in $L_{a}^{2}(\rho(M)) / L_{a}^{2}(N)$ for every $1 \leq i \leq n$, that is, there exist $\alpha_{0}^{(i)}, \alpha_{1}^{(i)}, \ldots, \alpha_{m(i)}^{(i)} \in \boldsymbol{C}$ with $\alpha_{m(i)}^{(i)} \neq 0$ such that $\sum_{j=0}^{m(i)} \alpha_{j}^{(i)}\left[f_{i} z^{j}\right]=0$. Put $g_{i}=\sum_{j=0}^{m(i)} \alpha_{j}^{(i)} f_{i} z^{j}$ and $h_{i}=\sum_{j=0}^{m(i)} \alpha_{j}^{(i)} z^{j}$. Then $g_{i}=f_{i} h_{i}$, and so $f_{i}$ is extended analytically on $N-h_{i}^{-1}(0)$, since $g_{i}$ is in $L_{a}^{2}(N)$, where

Note that

$$
h_{i}^{-1}(0)=\left\{z \in N-\rho(M) \mid h_{i}(z)=0\right\} .
$$

$$
N-\rho(M)=N \cap \partial(\rho(M))
$$

holds, since $\rho(M) \subset N \subset \overline{\rho(M)}$. Denote the extended function of $f_{i}$ also by $f_{i}$. Since $L_{a}^{2}(N)$ is generated by $f_{1}, \ldots, f_{n}$ and $L_{a}^{2}(\rho(M))$, and since $\bigcup_{i=1}^{n} h_{i}^{-1}(0)$ is a finite set, we see by the definition of the essential boundary that

$$
N \cap \partial_{e}(\rho(M)) \subset \bigcup_{i=1}^{n} h_{i}^{-1}(0) .
$$

Next we show that

$$
\bigcup_{i=1}^{n} h_{i}^{-1}(0) \subset N \cap \partial_{r}(\rho(M))
$$

Let $p \in \bigcup_{i=1}^{n} h_{i}^{-1}(0)$. We show that every $f_{i}$ is extended analytically at $p$. Put and fix $1 \leq$ $i \leq n$. If $h_{i}(p) \neq 0$, then $f_{i}$ is analytic at $p$, since $f_{i}=g_{i} / h_{i}$ near $p$. Suppose that $h_{i}(p)=0$. Then $f_{i}$ is analytic at $p$ or $p$ is a pole of $f_{i}$ since $g_{i}=f_{i} h_{i}$ is analytic at $p$. We show that $p$ cannot be a pole of $f_{i}$. Suppose that $p$ is a pole of $f_{i}$. Then $p \in N \cap \partial_{e}(\rho(M))$ by the 
definition of the essential boundary. Since $N \cap \partial_{e}(\rho(M)) \subset \bigcup_{i=1}^{n} h_{i}^{-1}(0)$ and $\bigcup_{i=1}^{n} h_{i}^{-1}(0)$ is a finite set, there exists an $r>0$ such that

$$
\{z \in \boldsymbol{C}|| z-p \mid<r\} \subset N
$$

and

$$
\{z \in C|| z-p \mid<r\} \cap N \cap \partial_{e}(\rho(M))=\{p\} .
$$

Hence we have

$$
\{z \in \partial(\rho(M))|| z-p \mid<r\}=\{p\} \cup\left\{z \in \partial_{r}(\rho(M))|| z-p \mid<r\right\} .
$$

By Proposition 2 in [1], $\partial_{r}(\rho(M))$ has zero area measure, and hence we have that $\{z \in$ $\partial(\rho(M))|| z-p \mid<r\}$ has zero area measure. On the other hand, we see that

$$
\{z \in \boldsymbol{C}|| z-p \mid<r\}-\{z \in \partial(\rho(M))|| z-p \mid<r\} \subset \rho(M),
$$

since $\rho(M) \subset N \subset \overline{\rho(M)}$. Since $f_{i} \in L_{a}^{2}(\rho(M))$, we have

$$
\begin{aligned}
\infty>\int_{\rho(M)}\left|f_{i}\right|^{2} d m & \geq \int_{\{z \in \boldsymbol{C}-\partial(\rho(M))|| z-p \mid<r\}}\left|f_{i}\right|^{2} d m \\
& =\int_{\{z \in \boldsymbol{C}|| z-p \mid<r\}}\left|f_{i}\right|^{2} d m,
\end{aligned}
$$

for $\{z \in \partial(\rho(M))|| z-p \mid<r\}$ has zero area measure, which is a contradiction since

$$
\int_{\{z \in \boldsymbol{C}|| z-p \mid<r\}}\left|f_{i}\right|^{2} d m=\infty,
$$

for $p$ is a pole of $f_{i}$. Thus we see that $f_{i}$ is extended analytically at $p$. It follows that every $f_{1}, \ldots, f_{n}$ is extended analytically at $p$, and hence

$$
\bigcup_{i=1}^{n} h_{i}^{-1}(0) \subset N \cap \partial_{r}(\rho(M)),
$$

since $L_{a}^{2}(\rho(M))$ is generated by $f_{1}, \ldots, f_{n}$ and $L_{a}^{2}(N)$.

Thus we have

$$
N \cap \partial_{e}(\rho(M)) \subset \bigcup_{i=1}^{n} h_{i}^{-1}(0) \subset N \cap \partial_{r}(\rho(M))
$$

Hence we see that

$$
N \cap \partial_{e}(\rho(M))=\emptyset,
$$

and so that

$$
N-\rho(M) \subset \partial_{r}(\rho(M)),
$$

since $\rho(M) \subset N \subset \overline{\rho(M)}$. 


\section{Some discussions on Toeplitz algebras.}

LEMMA 5 ([1] Theorem 9). Let $M$ be a bounded, open, connected, non-empty subset of $\boldsymbol{C}$, and $\mathcal{J}(M)$ be the algebra generated by $\left\{T_{\varphi} \mid \varphi \in C(\bar{M})\right\}$. Then the commutator ideal of $\mathcal{J}(M)$ is $\mathcal{K}(M)$, the space of compact operators on $L_{a}^{2}(M)$. Furthermore $\mathcal{J}(M) / \mathcal{K}(M)$ and $C\left(\partial_{e} M\right)$ are isometrically isomorphic $C^{*}$-algebras with an isomorphism that maps $T_{\varphi}+\mathcal{K}(M)$ to $\left.\varphi\right|_{\partial_{e} M}$ for each $\varphi \in C(\bar{M})$, where $\partial_{e} M$ denotes the Bergman essential boundary of $M$, consisting of all points of $\partial M$ which are not removable with respect to $L_{a}^{2}(M)$.

Definition 2. Let $M$ be a bounded domain in the complex plane, whose boundary $\partial M$ consists of a finitely many simple closed smooth analytic curves $\Gamma_{i}, i=1, \ldots, n$, where $\Gamma_{i}$ are positively oriented with respect to $M$ and $\Gamma_{i} \cap \Gamma_{j}=\emptyset$ if $i \neq j$. Then $M$ is said to be a finitely connected domain.

LEMMA 6 ([9]). Suppose that $M$ is a domain obtained from the unit disc by removing a finite number of disjoint discs. Then for $\varphi \in C(\bar{M})$, if $T_{\varphi}$ is Fredholm, then

$$
\text { Ind } T_{\varphi}=- \text { degree }(\varphi, 0) \text {, }
$$

where degree $(\varphi, 0)$ denotes the topological degree of $\varphi$ with respect to 0 .

LEMMA 7. Suppose that $M$ is a finitely connected domain in the complex plane. Then for $\varphi \in C(\bar{M})$, if $T_{\varphi}$ is Fredholm, then

$$
\text { Ind } T_{\varphi}=- \text { degree }(\varphi, 0) \text {. }
$$

PROOF. This is a simple consequence of Lemma 6.

The following Lemma 8 is a special case of a folklore theorem (see for instance, pp. 29 to 30 of [10]).

LEMma 8. Suppose that $M$ and $N$ are two closed subsets of the complex plane. Then $C(M)$ and $C(N)$ are algebraically isomorphic, denoted by $C(M) \cong C(N)$, if and only if $M$ and $N$ are homeomorphic.

Now, let $M$ be a finitely connected domain whose boundary $\partial M$ consists of simple closed smooth analytic curves $\Gamma_{i}, i=1, \ldots, n$, where $\Gamma_{i}$ are positively oriented with respect to $M$ and $\Gamma_{i} \cap \Gamma_{j}=\emptyset$ if $i \neq j$. Also, let $N$ be another finitely connected domain whose boundary $\partial N$ consists of simple closed smooth analytic curves $\widetilde{\Gamma}_{j}, j=1, \ldots, m$, where $\widetilde{\Gamma}_{j}$ are positively oriented with respect to $N$ and $\widetilde{\Gamma_{i}} \cap \widetilde{\Gamma}_{j}=\emptyset$ if $i \neq j$. Thus $\partial M=\bigcup_{i=1}^{n} \Gamma_{i}$ and $\partial N=\bigcup_{j=1}^{m} \widetilde{\Gamma_{j}}$. Furthermore, we have

$$
C(\partial M) \cong \bigoplus_{i=1}^{m} C\left(\Gamma_{i}\right), \quad C(\partial N) \cong \bigoplus_{j=1}^{m} C\left(\widetilde{\Gamma}_{j}\right)
$$

and

$$
C(\partial M, \boldsymbol{T}) \cong \bigoplus_{i=1}^{m} C\left(\Gamma_{i}, \boldsymbol{T}\right), \quad C(\partial N, \boldsymbol{T}) \cong \bigoplus_{j=1}^{m} C\left(\widetilde{\Gamma}_{j}, \boldsymbol{T}\right)
$$


It follows from these that
(1) $\pi^{1}(\partial M) \cong \sum_{i=1}^{n} \pi^{1}\left(\Gamma_{i}\right)=\overbrace{\boldsymbol{Z} \oplus \cdots \oplus \boldsymbol{Z}}^{n \text { times }}$,
(2) $\pi^{1}(\partial N) \cong \sum_{j=1}^{m} \pi^{1}\left(\widetilde{\Gamma}_{i}\right)=\overbrace{\boldsymbol{Z} \oplus \cdots \oplus \boldsymbol{Z}}^{m \text { times }}$

where $\pi^{1}(\partial M)$ denotes the first cohomotopy group of $\partial M$ which is defined by

$\pi^{1}(\partial M)=\{[f] \mid f \in C(\partial M, T)$ and $[f]$ is the homotopy equivalent class of $f\}$, and

(3) $\quad K_{0}(C(\partial M)) \cong \bigoplus_{i=1}^{n} K_{0}\left(C\left(\Gamma_{i}\right)\right) \cong \overbrace{\boldsymbol{Z} \oplus \cdots \oplus \boldsymbol{Z}}^{n \text { times }}$,

(4) $K_{0}(C(\partial N)) \cong \bigoplus_{j=1}^{m} K_{0}\left(C\left(\Gamma_{j}\right)\right) \cong \overbrace{\boldsymbol{Z} \oplus \cdots \oplus \boldsymbol{Z}}^{m \text { times }}$,

where $K_{0}(C(\partial M))$ is the $K_{0}$-group of $C(\partial M)$ (see for instance, the definition of [11]). Hence $K_{0}(C(\partial M)) \cong K_{0}(C(\partial N))$ if and only if $\pi^{1}(\partial M) \cong \pi^{1}(\partial N)$, which holds if and only if $\partial M$ and $\partial N$ are homeomorphic.

By Lemma 5, we have the following exact sequences:

$$
\begin{gathered}
0 \longrightarrow \mathcal{K}(M) \stackrel{i_{1}}{\longrightarrow} \mathcal{J}(M) \stackrel{\rho_{1}}{\longrightarrow} C(\partial M) \longrightarrow 0, \\
0 \longrightarrow \mathcal{K}(N) \stackrel{i_{1}}{\longrightarrow} \mathcal{J}(N) \stackrel{\rho_{1}}{\longrightarrow} C(\partial N) \longrightarrow 0,
\end{gathered}
$$

from which we obtain the exact sequences:

$$
\begin{gathered}
K_{0}(\mathcal{K}(M)) \stackrel{i_{1}^{*}}{\longrightarrow} K_{0}(\mathcal{J}(M)) \stackrel{\rho_{1}^{*}}{\longrightarrow} K_{0}(C(\partial M)), \\
K_{0}(\mathcal{K}(N)) \stackrel{i_{1}^{*}}{\longrightarrow} K_{0}(\mathcal{J}(N)) \stackrel{\rho_{1}^{*}}{\longrightarrow} K_{0}(C(\partial N)) .
\end{gathered}
$$

Thus, we may prove easily the following

THEOREM 9. Suppose that $M$ and $N$ are finitely connected domains. If

$$
K_{0}(\mathcal{J}(M)) \cong K_{0}(\mathcal{J}(N)),
$$

then the following hold:

(1) $\pi^{1}(\partial M) \cong \pi^{1}(\partial N)$.

(2) $C(\partial M) \cong C(\partial N)$.

(3) $\partial M$ and $\partial N$ are homeomorphic.

Proof. By Lemma 8 and the discussions above, we need only to prove (3). Let $K_{1}(A)$ be the $K_{1}$-group of a $C^{*}$-algebra $A$ (see for instance, the definition of [11]). By the following six-term exact sequences

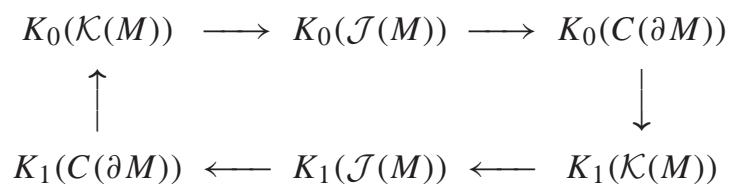


and

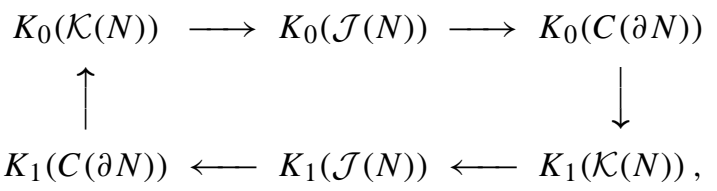

we obtain two exact sequences:

$$
\begin{gathered}
\boldsymbol{Z} \stackrel{i_{1}^{*}}{\longrightarrow} K_{0}(\mathcal{J}(M)) \stackrel{\rho_{1}^{*}}{\longrightarrow} K_{0}(C(\partial M)) \longrightarrow 0, \\
\boldsymbol{Z} \stackrel{i_{2}^{*}}{\longrightarrow} K_{0}(\mathcal{J}(N)) \stackrel{\rho_{2}^{*}}{\longrightarrow} K_{0}(C(\partial N)) \longrightarrow 0,
\end{gathered}
$$

since $K_{0}(\mathcal{K}(M)) \cong K_{0}(\mathcal{K}(N)) \cong Z$ and $K_{1}(\mathcal{K}(M))=K_{1}(\mathcal{K}(N))=0$.

Assume that $\alpha$ is the isomorphism from $K_{0}(\mathcal{J}(M))$ onto $K_{0}(\mathcal{J}(N))$. Then there exists a homomorphism $\beta$ from $K_{0}(C(\partial M))$ to $K_{0}(C(\partial N))$ such that the following diagram is commutative

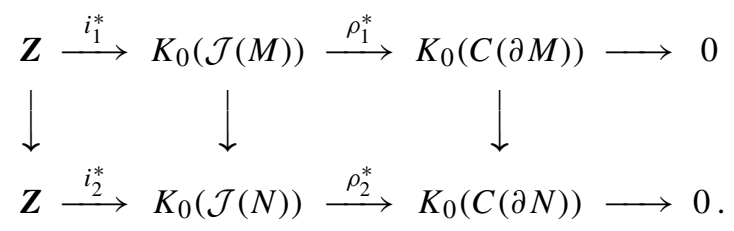

It is now routine to check that $\beta$ is an isomorphism, and hence $K_{0}(C(\partial M)) \cong K_{0}(C(\partial N))$. Thus $\partial M$ and $\partial N$ are homeomorphic.

Theorem 9 implies that if the number of holes in $M$ does not equal that in $N$, then $K_{0}(\mathcal{J}(M))$ and $K_{0}(\mathcal{J}(N))$ are never isomorphic. This is different from the case of algebras of continuous functions. For example, $K_{0}(C(\overline{\boldsymbol{D}}))=K_{0}(C(\boldsymbol{T}))=\boldsymbol{Z}$, where $\boldsymbol{T}$ denotes the topological boundary of the unit disc $\boldsymbol{D}$.

REMARK. If $M$ and $N$ are bounded connected domains, and

$$
K_{0}(\mathcal{J}(M)) \cong K_{0}(\mathcal{J}(N)),
$$

then we obtain a commutative diagram

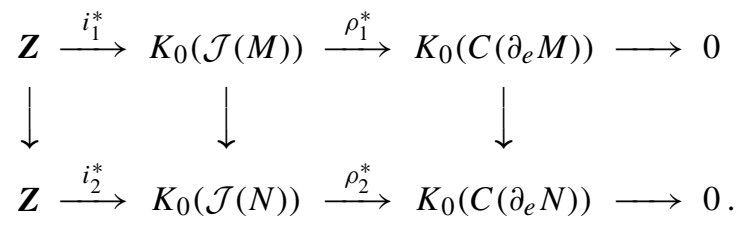

Furthermore, $K_{0}\left(C\left(\partial_{e} M\right)\right) \cong K_{0}\left(C\left(\partial_{e} N\right)\right)$. However we do not know whether this implies that $\partial_{e} M$ and $\partial_{e} N$ are homeomorphic.

Finally, we discuss the isomorphism between two Toeplitz algebras. 
THEOREM 10. Suppose $M$ and $N$ are domains. If $\mathcal{J}(M) \stackrel{\alpha}{\cong} \mathcal{J}(N)$, then there is $a$ homeomorphism $\rho$ from $\partial_{e} M$ onto $\partial_{e} N$ satisfying Ind $T_{\lambda}=$ Ind $S_{\lambda}$ for any $\lambda \notin \partial_{e} N, T_{\lambda} \in$ $\xi_{M}^{-1}(\rho-\lambda)$ and $S_{\lambda} \in \xi_{N}^{-1}\left(\varphi_{0}-\lambda\right)$ such that

$$
\xi_{N} \hat{\alpha} \xi_{M}^{-1}(\varphi)=C_{\rho^{-1}}(\varphi) \text { for each } \varphi \in C\left(\partial_{e} M\right),
$$

where $\hat{\alpha}([T])=[\alpha(T)]$ for $[T] \in \mathcal{J}(M) / \mathcal{K}(M), \varphi_{0}(z)=z, \xi_{M}$ is the isomorphism from $\mathcal{J}(M) / \mathcal{K}(M)$ onto $C\left(\partial_{e} M\right)$, and $\xi_{N}$ is the isomorphism from $\mathcal{J}(N) / \mathcal{K}(N)$ onto $C\left(\partial_{e} N\right)$.

Conversely, if $\rho$ is a homeomorphism from $\partial_{e} M$ onto $\partial_{e} N$ with Ind $T_{\lambda}=$ Ind $S_{\lambda}$ for any $\lambda \notin \partial_{e} N, T_{\lambda} \in \xi_{M}^{-1}(\rho-\lambda)$ and $S_{\lambda} \in \xi_{N}^{-1}\left(\varphi_{0}-\lambda\right)$, then there is an isomorphism from $\mathcal{J}(M)$ onto $\mathcal{J}(N)$ such that $(*)$ holds.

Proof. Suppose that $\alpha: \mathcal{J}(M) \rightarrow \mathcal{J}(N)$ is an isomorphism. Then there is a unitary operator $U$ from $L_{a}^{2}(N)$ onto $L_{a}^{2}(M)$ such that $\alpha(T)=U^{*} T U$ for $T \in \mathcal{J}(M)$, since $\mathcal{K} \subset$ $\mathcal{J}(M)$ (cf. Douglas [12]). Note that $\mathcal{J}(M) / \mathcal{K}(M) \stackrel{\xi_{M}}{\cong} C\left(\partial_{e} M\right)$ and $\mathcal{J}(N) / \mathcal{K}(N) \stackrel{\xi_{N}}{\cong} C\left(\partial_{e} N\right)$. We then see that $\mathcal{J}(M) / \mathcal{K}(M) \cong \mathcal{J}(N) / \mathcal{K}(N)$ if and only if $C\left(\partial_{e} M\right) \cong C\left(\partial_{e} N\right)$. Thus there is a homeomorphism $\rho$ from $\partial_{e} M$ onto $\partial_{e} N$ such that

$$
\xi_{N}(\hat{\alpha}([T]))=\xi_{M}([T]) \circ \rho^{-1} .
$$

Furthermore, we have

$$
\xi_{N} \hat{\alpha} \xi_{M}^{-1}(\varphi)=C_{\rho^{-1}}(\varphi) \text { for each } \varphi \in C\left(\partial_{e} M\right) .
$$

Set $\varphi(z)=\rho(z)$. Then we see that

$$
\xi_{N} \hat{\alpha} \xi_{M}^{-1}(\rho)=\varphi_{0}
$$

Hence Ind $T_{\lambda}=$ Ind $S_{\lambda}$ for any $\lambda \notin \partial_{e} N, T_{\lambda} \in \xi_{M}^{-1}(\rho-\lambda)$ and $S_{\lambda} \in \xi_{N}^{-1}\left(\varphi_{0}-\lambda\right)$ by Lemma 7.

Conversely, assume that $\rho$ is a homeomorphism from $\partial_{e} M$ onto $\partial_{e} N$ with Ind $T_{\lambda}=$ Ind $S_{\lambda}$ for any $\lambda \notin \partial_{e} N, T_{\lambda} \in \xi_{M}^{-1}(\rho-\lambda)$ and $S_{\lambda} \in \xi_{N}^{-1}\left(\varphi_{0}-\lambda\right)$. Noting that $\sigma_{e}\left(T_{\lambda}\right)=$ $(\rho-\lambda)\left(\partial_{e} M\right)=\partial_{e} N-\lambda=\sigma_{e}\left(S_{\lambda}\right)$, by BDF theorem, we see that $T_{\lambda}$ and $S_{\lambda}$ are essential unitary equivalent. Namely, there exists a unitary operator $U: L_{a}^{2}(M) \rightarrow L_{a}^{2}(N)$ such that

$$
T_{\lambda}-U^{*} S_{\lambda} U \in \mathcal{K}\left(L_{a}^{2}(M)\right) .
$$

Denote by $\mathcal{A}_{\rho}$ the subalgebra of $C\left(\partial_{e} M\right)$ which is generated by $1, \rho$ and $\bar{\rho}$. By StoneWeierstrass theorem, we know that $\mathcal{A}_{\rho}=C\left(\partial_{e} M\right)$. Since $T_{\varphi} T_{\psi}-T_{\varphi \psi}$ is compact for any $\varphi, \psi \in C\left(\partial_{e} M\right)$ or $\varphi, \psi \in C\left(\partial_{e} N\right)$, it follows that for arbitrary $\varphi \in C\left(\partial_{e} N\right), T \in \xi_{M}^{-1}(\varphi \circ \rho)$ and $S \in \xi_{N}^{-1}(\varphi)$, we have $T-U^{*} S U \in \mathcal{K}\left(L_{a}^{2}(M)\right)$. It is clear that $\alpha(S)=U^{*} S U \in \mathcal{J}(M)$, $S \in \mathcal{J}(N)$, is an isomorphism and (*) holds.

LeMma 11 ([1], Proposition 3). If $M$ is a bounded domain in the complex plane $\boldsymbol{C}$. Then $M \cup \partial_{r} M$ is an open subset of $\boldsymbol{C}$.

LEMMA 12. If $M$ is a bounded domain in $C$, then

$$
\partial\left(M \cup \partial_{r} M\right)=\partial_{e} M .
$$


PROOF. First, we prove that $\partial_{r}\left(M \cup \partial_{r} M\right)=\varnothing$. Indeed, if $z \in \partial_{r}\left(M \cup \partial_{r} M\right)$, then there exists a neighborhood $V(z)$ of $z$ such that each $f \in L_{a}^{2}\left(M \cup \partial_{r} M\right)$ may be extended to an analytic function defined on $M \cup \partial_{r} M \cup V(z)$. Since $\partial\left(M \cup \partial_{r} M\right) \subset \partial M$, we see that $z \in \partial_{r} M \subset M \cup \partial_{r} M$. This contradiction shows that $\partial_{r}\left(M \cup \partial_{r} M\right)=\emptyset$. Thus $\partial\left(M \cup \partial_{e} M\right)=$ $\partial_{e}\left(M \cup \partial_{r} M\right)$. Furthermore, $\partial_{e}\left(M \cup \partial_{r} M\right) \subset \partial_{e} M$.

Now, if $z \in \partial_{e} M$, then for any neighborhood $U(z)$ of $z,(U(z)-\{z\}) \cap M \neq \varnothing$ and $U(z) \cap(\boldsymbol{C}-M) \neq \varnothing$, so that $(U(z)-\{z\}) \cap\left(M \cup \partial_{r} M\right) \neq \emptyset$. Since $z \notin \partial_{r} M$, we see that $z \notin M \cup \partial_{r} M$. Consequently, $z \in \partial\left(M \cup \partial_{r} M\right)$.

By Lemma 12, if $\rho: \partial_{e} M \rightarrow \partial_{e} N$ is a homeomorphism and $\lambda \notin \partial_{e} N$, then we may define the degree of $\rho$ with respect to $\lambda$ to be

$$
\operatorname{degree}(\rho, \lambda)=\operatorname{degree}\left(\hat{\rho}, M \cup \partial_{r} M, \lambda\right),
$$

where $\hat{\rho}$ is an any continuous extension of $\rho$ on $M \cup \partial_{r} M$. Since degree $\left(\hat{\rho}, M \cup \partial_{r} M, \lambda\right)$ depends only on the boundary values of $\hat{\rho}$, we see that the definition is well-defined.

However, we do not know whether Ind $T_{\lambda}=-\operatorname{degree}(\rho, \lambda)$ for any $\lambda \notin \partial_{e} N$ and $T_{\lambda} \in$ $\xi_{M}^{-1}(\rho-\lambda)$. If $M$ and $N$ are finitely connected domains, then the index formula holds by Lemma 7. Thus we have the following

COROLlary. Suppose that $M$ and $N$ are finitely connected domains such that $\partial M$ and $\partial N$ have no isolated points. If $\mathcal{J}(M) \stackrel{\alpha}{\cong} \mathcal{J}(N)$, then there is a homeomorphism $\rho$ from $\partial M$ onto $\partial N$ satisfying degree $(\rho, \lambda)=\operatorname{degree}\left(\varphi_{0}, \lambda\right)$ for any $\lambda \notin \partial N$ such that

$$
\xi_{N} \hat{\alpha} \xi_{M}^{-1}(\varphi)=C_{\rho^{-1}}(\varphi) \text { for each } \varphi \in C(\partial M),
$$

where $\hat{\alpha}([T])=[\alpha(T)]$ for $[T] \in \mathcal{J}(M) / \mathcal{K}(M), \varphi_{0}(z)=z, \xi_{M}$ is the isomorphism from $\mathcal{J}(M) / \mathcal{K}(M)$ onto $C(\partial M)$, and $\xi_{N}$ is the isomorphism from $\mathcal{J}(N) / \mathcal{K}(N)$ onto $C(\partial N)$.

Conversely, if $\rho$ is a homeomorphism from $\partial M$ onto $\partial N$ with degree $(\rho, \lambda)=$ degree $\left(\varphi_{0}, \lambda\right)$ for any $\lambda \notin \partial N$, then there is an isomorphism from $\mathcal{J}(M)$ onto $\mathcal{J}(N)$ such that $(* *)$ holds.

Proof. By the assumption, we see that $\partial_{r} M=\emptyset$ and $\partial_{r} N=\emptyset$. The Corollary is proved by Theorem 10 .

\section{REFERENCES}

[ 1 ] S. AXler, J. B. Conway And G. MCDonald, Toeplitz operators on Bergmann spaces, Canad. J. Math. 34 (1982), 466-483.

[2] J. A. Cima, J. Thomson And W. Wogen, On some properties of composition operators, Indiana Univ. Math. 24 (1974), 215-220.

[ 3 ] O. HATORI, Fredholm composition operators on spaces of holomorphic functions, Integral Equations Operator Theory 18 (1994), 202-210.

[ 4 ] G. F. CAO AND S. H. SUn, On composition operators on $H^{p}$-spaces in several variables, Acta Math. Sinica (N. S.) 13 (1997) 281-288.

[ 5 ] D. Bourdon, Fredholm multiplication and composition operators on the Hardy spaces, Integral Equations Operators Theory 13 (1990), 607-610. 
[ 6 ] R. K. Singh And T. Veluchamy, Non-atomic measure spaces and Fredholm composition operators, Acta Sci. Math. 51 (1987), 461-465.

[ 7 ] H. TAKAGI, Fredholm weighted composition operators, Integral Equations Operator Theory 16 (1993), 267276.

[ 8 ] I. Mihaila, Composition operators on Riemann Surfaces, Contemp. Mathematics 213 (1998), 145-155.

[ 9 ] G. F. CAO AND X. F. WANG, Operators on Bergmann spaces for Riemann surfaces. preprint.

[10] A. BRowDER, Introduction to Function Algebras, Benjamin, New York, 1968.

[11] M. Rordam, F. Larsen and N. Laustsen, An introduction to $K$-theory for $C^{*}$-algebras, Cambridge University Press, Cambrige, 2000.

[12] R. G. Douglas, Banach algebra techniques in operator theory, Pure and Applied Mathematics, Vol. 49, Academic Press, New York-London, 1972.

DEPARTMENT OF MATHEMATICS

GUANGZHOU UNIVERSITY

GUANGZHOU 510405

PEOPLE'S REPUBLIC OF CHINA

E-mail address: guangfucao@163.com 